\title{
BMJ Open Evaluating the impact of type 2 diabetes mellitus on CYP450 metabolic activities: protocol for a case-control pharmacokinetic study
}

\author{
Sophie Gravel, ${ }^{1,2}$ Jean-Louis Chiasson, ${ }^{2,3,4}$ Suzanne Dallaire, ${ }^{2}$ Jacques Turgeon, ${ }^{1,2}$ \\ Veronique Michaud ${ }^{1,2}$
}

To cite: Gravel S, Chiasson J-L, Dallaire $\mathrm{S}$, et al. Evaluating the impact of type 2 diabetes mellitus on CYP450 metabolic activities: protocol for a case-control pharmacokinetic study. BMJ Open 2018;8:e020922. doi:10.1136/ bmjopen-2017-020922

- Prepublication history for this paper is available online. To view these files, please visit the journal online (http://dx.doi. org/10.1136/bmjopen-2017020922).

Received 1 December 2017 Accepted 19 December 2017

Check for updates

${ }^{1}$ Faculty of Pharmacy, Université de Montréal, Montreal, Canada

${ }^{2}$ Centre de Recherche du Centre Hospitalier de l'Université de Montréal (CRCHUM), Montreal, Canada

${ }^{3}$ Centre Hospitalier de

I'Université de Montréal (CHUM), Montreal, Canada

${ }^{4}$ Faculty of Medicine, Université de Montréal, Montreal, Canada

Correspondence to

Dr Veronique Michaud; v.michaud@umontreal.ca

\section{ABSTRACT}

Introduction Diabetes affects more than $9 \%$ of the adult population worldwide. Patients with type 2 diabetes mellitus (T2DM) show variable responses to some drugs which may be due, in part, to variability in the functional activity of drug-metabolising enzymes including cytochromes P450 (CYP450s). CYP450 is a superfamily of enzymes responsible for xenobiotic metabolism. Knowledge must be gained on the impact of T2DM and related inflammatory processes on drug metabolism and its consequences on drug response. The aim of this study is to characterise the activity of CYP1A2, CYP2B6, CYP2C9, CYP2C19, CYP2D6, CYP2E1 and CYP3A4/5 in T2DM versus non-T2DM subjects following the administration of a cocktail of probe drug substrates.

Methods and analysis This single-centre clinical study proposes the first detailed characterisation of T2DM impacts on major CYP450 drug-metabolising enzyme activities. We intend to recruit 42 patients with controlled T2DM (A1C $\leq 7 \%)$, 42 patients with uncontrolled T2DM (A1C $>7 \%$ ) and 42 non-diabetic control subjects. The primary objective is to determine and compare major CYP450 activities in patients with T2DM versus nondiabetic subjects by dosing in plasma and urine probe drug substrates and metabolites following the oral administration of a drug cocktail: caffeine (CYP1A2), bupropion (CYP2B6), tolbutamide (CYP2C9), omeprazole (CYP2C19), dextromethorphan (CYP2D6), chlorzoxazone (CYP2E1) and midazolam (CYP3A4/5). Secondary objectives will evaluate the influence of variables such as glycaemia, insulinaemia, genetic polymorphisms and inflammation. The value of an endogenous biomarker of CYP3A activity is also evaluated. The first patient was recruited in May 2015 and patients will be enrolled up to completion of study groups.

Ethics and dissemination Approval was obtained from the ethic review board of the CHUM research centre (Montreal, Canada).

Trial registration number NCT02291666.

\section{INTRODUCTION}

The increasing prevalence of diabetes mellitus, a pathology which is associated with numerous comorbidities, is of major concern

\section{Strengths and limitations of this study}

- Study population including a control group of non-type 2 diabetes mellitus (T2DM) subjects and patients with T2DM (patients with T2DM will be stratified according to their glycaemic control) will provide valuable information to better understand T2DM impacts on drug metabolism and to dissect the role of glycaemia versus inflammatory factors.

- The use of a validated cocktail of seven probe substrates along with highly precise, fast and robust quantification methods will permit the simultaneous determination of phenotypes for seven major cytochromes P450 (CYP450) isozymes involved in drug metabolism: CYP1A2, CYP2B6, CYP2C9, CYP2C19, CYP2D6, CYP2E1 and CYP3A4/5.

- An exhaustive data collection and review of medical and pharmacological records for all participants will permit evaluation of covariables and investigation of mechanisms underlying expected modulation of CYP450 isozyme activities.

- The use of endogenous $4 \llbracket$-hydroxycholesterol plasma concentrations will be assessed as a putative biomarker of CYP3A4/5 activity.

- Study population is limited to individuals with a body mass index $\leq 35 \mathrm{~kg} / \mathrm{m}^{2}$ which, although reducing confounding factors, prevents the extrapolation of our results to morbid obese individuals.

due to its important burden on society and healthcare systems. ${ }^{12}$ In 2014, diabetes was estimated to affect more than $9 \%$ of the worldwide adult population. ${ }^{3}$ Type 2 diabetes mellitus (T2DM), the most common form of diabetes, is characterised by insulin resistance, an incapacity of the body to respond adequately to insulin action, and by beta cell dysfunction, an inability to respond to a glucose challenge by an appropriate insulin release. The resulting elevated blood glucose levels can lead to the development of costly and serious complications and comorbidities such as neuropathy, nephropathy, 
retinopathy, heart disease and an elevated incidence of stroke. ${ }^{4-6}$ In 2013, diabetes mellitus was the seventh leading cause of death in the USA. ${ }^{7}$

Prevention and treatment of T2DM, its complications and comorbidities often require the use of multiple drugs to address these issues. Polypharmacy, per se, increases the risk of multidrug interactions leading to adverse drug reactions (ADRs) which account for about $6.5 \%$ of all hospital admissions. ${ }^{8}$ Furthermore, the risk of ADRs and treatment failure appears to be increased in patients with T2DM. Indeed, it has been previously reported that patients with T2DM tend to show highly variable responses to different drugs; while some patients are resistant to some drugs, some are more sensitive to other drugs (eg, warfarin, clopidogrel, cyclosporin, tacrolimus and antihypertensive agents) ${ }^{9-14}$

Cytochrome P450 (CYP450) is a superfamily of enzymes responsible for drug metabolism that represents a major source of variability in drug pharmacokinetics and response. CYP450 metabolising activities can be regulated by many intrinsic and extrinsic factors such as genetics and environment. For instance, proinflammatory cytokines have been shown to decrease CYP3A and CYP2C activities. ${ }^{15-17}$ Thereby, chronic inflammatory diseases such as T2DM can impact CYP450 isoenzymes and modulate the patients' response to drugs. ${ }^{18}$ The impact of T2DM on drug-metabolising enzymes could be an important factor to consider clinically to improve treatment of patients with T2DM by managing efficiently the use of multiple concomitant drugs to reduce ADRs or to optimise efficacy. To reach an optimised drug utilisation, knowledge has to be gained on the influence of T2DM per se on drug metabolism.

Our hypothesis is that T2DM and related inflammatory processes alter activities of CYP450s involved in drug disposition which may explain variability in drug response. The specific aims are (1) to determine CYP450 phenotypes in patients with T2DM versus non-diabetic subjects following a single oral administration of a cocktail of CYP450 probe drugs and (2) to compare CYP450 phenotypes according to the glycaemic control.

\section{METHODS AND ANALYSIS}

This clinical study is an open-label explorative pharmacokinetic research investigating the effects of T2DM on major CYP450 activities, namely, CYP1A2, CYP2B6, CYP2C9, CYP2C19, CYP2D6, CYP2E1 and CYP3As. Study protocol (CHUM's Trial \#14.066) was registered at the US National Institutes of Health website (http://www.clinicaltrial.gov; NCT02291666).

\section{Study population}

A total of 126 participants, gender-matched between groups, are to be recruited from the Diabetes Registry at the CRCHUM outpatient clinic to constitute three study groups.

\begin{tabular}{|c|c|}
\hline $\begin{array}{l}\text { Inclusion } \\
\text { criteria }\end{array}$ & $\begin{array}{l}\text { Male and female (non-pregnant) aged } \\
18 \text { years and over } \\
\text { Glomerular filtration rate }>50 \mathrm{~mL} / \mathrm{min} / 1.73 \mathrm{~m}^{2} \\
\text { Acceptable hepatic function (alanine } \\
\text { transaminase and aspartate transaminase } \\
\text { levels below three times the normal range) } \\
\text { - Body mass index } \leq 35 \mathrm{~kg} / \mathrm{m}^{2} \\
\text { - Non-smoker for at least } 2 \text { months }\end{array}$ \\
\hline $\begin{array}{l}\text { Exclusion } \\
\text { criteria }\end{array}$ & $\begin{array}{l}\text { Use of: } \\
\text { - Antibiotics, antivirals, antiretrovirals, } \\
\text { monoamine oxidase inhibitors, } \\
\text { immunosuppressants, interferons and } \\
\text { antibodies } \\
\text { - Cytochrome P450 (CYP450) inducers (eg, } \\
\text { carbamazepine, phenobarbital, phenytoin, } \\
\text { rifampicin, St. John's wort) } \\
\text { - CYP450 inhibitors (eg, amiodarone, } \\
\text { fluvoxamine, fluoxetine, verapamil, } \\
\text { terbinafine). } \\
\text { Uncontrolled thyroid function } \\
\text { Presence of an important inflammatory } \\
\text { condition } \\
\text { A diagnosed gastrointestinal pathology } \\
\text { Patients with an active cancer } \\
\text { Patients who underwent a transplant or a } \\
\text { bariatric surgery }\end{array}$ \\
\hline
\end{tabular}

A. Forty-two patientswith T2DM with good glycaemic control defined here as an $\mathrm{A} 1 \mathrm{C} \leq 7 \%$.

B. Forty-two patients with T2DM with inadequate glycaemic control defined here as an $\mathrm{A} 1 \mathrm{C}>7 \%$.

C. Forty-two non-diabetic participants serving as control.

Eligibility criteria include participants $\geq 18$ years old and with a body mass index (BMI) $\leq 35 \mathrm{~kg} / \mathrm{m}^{2}$. They have to be non-smoker for at least 2 months prior to study and to abstain from grapefruit juice within 2 weeks before drug administration. Exclusion criteria also include a review of all subjects' pharmacological record to control for the use of CYP450 inhibitor or inducer drugs. Detailed eligibility criteria are presented in table 1 . Written informed consent is to be obtained from all participants prior to any initiation of study procedure.

\section{Primary endpoints}

Our primary endpoint is to compare phenotypes for seven major CYP450 drug-metabolizing enzymes by calculating metabolic ratios in our study groups, that is, T2DM (A1C $\leq 7 \%$ and $\mathrm{A} 1 \mathrm{C}>7 \%$ ) and non-T2DM (control), by using well-characterized isoform selective orally administered probe substrates:

(1) a CYP450 probe drug cocktail (CRCHUM-MT cocktail) comprising:

- caffeine (Wake Ups $100 \mathrm{mg}$, Adem), CYP1A2;

- bupropion (Bupropion SR $100 \mathrm{mg}$, Sandoz), CYP2B6;

- tolbutamide (Orinase 250 mg, AA Pharma), CYP2C9; 
- omeprazole (APO-Omeprazole 20 mg, Apotex), CYP2C19;

- dextromethorphan (Bronchophan Forte DM $30 \mathrm{mg}$, Atlas Laboratories), CYP2D6;

- midazolam (Midazolam injection, USP, $2 \mathrm{mg}$, Fresenius Kabi), CYP3As

(2) chlorzoxazone alone (Acetazone Forte $250 \mathrm{mg}$, Rougier), CYP2E1.

All participants will be studied at the Phase 1 Unit at the CRCHUM starting at 07:00. Subsequent to cocktail administration, blood and urine samples will be collected at specified times (for 24 and 8 hours, respectively). All drug concentration-time data will be analysed by standard non-compartmental methods using Kinetica V.5.1 (Thermo Kinetica, Thermo Fisher Scientific, PA, USA). The area under the curve $0-8$ hour $\left(\mathrm{AUC}_{0-8}\right)$ will be obtained by use of the mixed log-linear trapezoidal rule enabling for interpolation and using the limit of quantification to compute last AUC triangle when the 8-hour time point is below the limit of quantification. Phenotypic indices of major CYP450 activities will be determined as previously validated using metabolic ratios of urinary or plasmatic concentrations. ${ }^{19-24}$ Each of our probe drugs are selective for a specific isoenzyme of the CYP450s and do not affect other isoenzyme activities. All probe drugs will be administered at the same time as a cocktail (07:00), except for chlorzoxazone which is administered separately in the evening of the experimental day with a urine sample collected overnight and blood sample collected the next morning.

\section{Secondary endpoints}

As a secondary endpoint, we will investigate the influence of different T2DM characteristics and covariates on the enzymatic activity of measured CYP450s activities. Principal variables investigated are as follows.

- Insulin resistance markers: insulin, blood glucose and glycated haemoglobin levels.

- Genetic polymorphisms associated with CYP450s under investigation which are known to modulate enzymatic activity.

- Proinflammatory cytokines: interferon gamma, interleukin 1-beta, interleukin 6 and tumor necrosis factor alpha levels.

- Covariates such as BMI, age, comedication and time since diagnosis.

Another exploratory endpoint will be to investigate the potential use of the plasma concentrations of cholesterol and $4 \beta$-hydroxycholesterol as an endogenous biomarker of CYP3A activity. Indeed, recent data suggest that this plasmatic ratio could be used as an endogenous marker of CYP3A activity in vivo. ${ }^{25} 26$

\section{Experimental protocol}

On experimental day, participants will be admitted to the clinical research unit of the CRCHUM at 07:00 after an overnight fast and will remain fasted until the 4-hour time point is collected. Serial blood samples will be drawn in
$6 \mathrm{~mL}$ K2-EDTA vacutainers via indwelling venous catheters immediately before (time 0 ) and at $0.33,0.66,1,1.5,2$, $3,4,6,8$ and 24 hours postadministration of the CYP450 probe drug cocktail for measurement of probe drugs and their metabolites. Blood samples will be kept on ice and centrifuged for $10 \mathrm{~min}$ at $4^{\circ} \mathrm{C}$ and $1500 \mathrm{~g}$ less than $15 \mathrm{~min}$ after collection. Plasma samples will then be stored in aliquots at $-80^{\circ} \mathrm{C}$ for later analysis. Urine will be collected prior to probe-drug administration (baseline) and over an 8-hour period after the cocktail administration. Urine will constantly be kept on ice and after measurement of total urine volume, aliquots will be stored at $-20^{\circ} \mathrm{C}$ until analysis. Patients will be discharged after the 8-hour urine and plasma sample collection. As an outpatient, on the same night, subjects will be asked to take an oral dose of chlorzoxazone prior to bedtime and collect all their urine for a minimal period of 8 hours after drug administration. This urine collection will be returned on the next morning to the research centre. After measurement of total urine volume, aliquots will be stored at $-20^{\circ} \mathrm{C}$ for later analysis.

Two blood samples collected in serum separator SST II tubes prior to dosing will be sent to the CHUM's biochemistry lab for insulin and glucose measurements. Furthermore, a blood sample will be collected in $6 \mathrm{~mL}$ K2-EDTA vacutainers prior to dosing for the measurement of inflammatory markers and plasmatic ratio

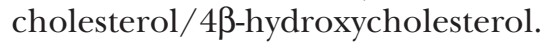

\section{Sample size calculations and statistical analyses}

Sample size was calculated for a significant difference of $30 \%$ between groups, with a power of $80 \%$ and an alpha value of 0.05 . The required sample size $(n=38$ subjects per group) was calculated to meet the primary objective considering the reported variances of CYP3A activities found in the literature (greatest changes have been observed with CYP3A4). Considering an anticipated drop-out rate of $10 \%, 42$ subjects per group will be recruited for a total of 126 participants.

Statistical analyses will be used to answer primary objective which consists of determining if T2DM disease modulates CYP450 activities. A stepwise statistical analysis will be performed: (1) each phenotypic probe will be compared between T2DM (including controlled and uncontrolled glycaemia) versus non-diabetic individuals and (2) T2DM group will be subcategorised based on $\mathrm{A} 1 \mathrm{C} \leq 7 \%$ versus A1C $>7 \%$ versus non-T2DM group. This aim will be tested for significance by performing an analysis of variance with a post hoc.

In a secondary analysis, the influence of covariables will be tested using multiple regression analyses. Different variables will be considered in the models: CYP450 genetic polymorphisms, BMI, insulin resistance markers, proinflammatory cytokines, age and time since diagnosis. Potential confounding variables will be defined according to: (1) variables associated with a specific CYP450 that have been reported in literature and (2) variables with a correlation coefficient above 0.2 or below -0.2 in T2DM groups versus non-diabetic group. 
Finally, plasma concentrations of the endogenous

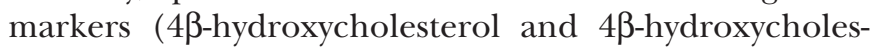
terol/cholesterol ratio) will be compared between the study groups and correlation will be established with activity observed for the probe substrate, midazolam, administered in the CRCHUM-MT cocktail.

\section{Data management}

In this project, confidentiality of collected data will be preserved by using an attributed identification number to all participants. Subjects' identities and numbers will only be accessible to designated investigators and all data will be treated in a non-nominal manner. All documentation related to study will be kept in locked drawers. DNA samples will solely be used for genetic analyses associated with CYP450s or other enzymes/transporters of drugs relevant for T2DM, associated comorbidities and drug response variability. It will not be used to serve any other purpose than this present study. DNA will be kept for a maximum period of 10 years before complete destruction (hydrochloric acid).

\section{Ethics and dissemination}

This study will be carried out in compliance with the Declaration of Helsinki and International Conference on Harmonisation Good Clinical Practice Guidelines. All participants will be informed of the objectives, risks and inconveniences of the trial and be provided with written information on the study, contact details of the principal investigators as well as copies of their signed consent/ assent forms. Subjects will be clearly informed of their right to withdraw from the study at any time during the process and this without consequences for their future care. Immediate intervention or treatment is available in case of an acute adverse event, for example, anaphylactic reaction.

A manuscript presenting and discussing all results of the primary objective as well as the covariables analyses will be submitted and published in a peer-reviewed journal. A second distinct manuscript addressing the use of an endogenous marker of CYP3A activity will be submitted in a peer-reviewed journal as well.

On completion of the trial and publication of the results, a request for experimental data can be submitted to principal investigator at the CRCHUM in Montreal, Canada.

\section{DISCUSSION}

Many drugs in addition to antidiabetic medications are prescribed to patients with T2DM to treat their numerous comorbidities. However, few information is available on the efficacy and dosing of these drugs in this subpopulation of patients. Indeed, during drug development processes, pharmacokinetic and pharmacodynamic studies of new medications for various indications are rarely tested in a population of patients with T2DM. Available data for this group of patients are often derived from secondary analyses of clinical trials. This research protocol will generate valuable results enabling a better understanding of factors affecting drug metabolism in vivo. New knowledge gained on metabolic capacity in patients with T2DM will improve our understanding of the underlying mechanisms responsible for the high interindividual variation in drug response observed in this group of patients. Thereby, it will provide new information to help clinicians improving use of drugs to reduce therapeutic failure and toxicity entailed by suboptimal or inappropriate use of pharmaceutical treatments.

Contributors VM conceived the study hypothesis, designed and wrote the trial protocol. JT and JLC provided revision of the proposed protocol. SG wrote the first draft of this manuscript. VM, JT and JLC revised and edited this study protocol article. All the authors contributed to the feasibility of the study and approved the final version of this manuscript.

Funding This work was supported by the Canadian Institutes of Health Research, grant number 299309. The funding sources for this trial had no role in the design of the study and will not have any role in the performance of the research.

Competing interests None declared.

Patient consent Obtained.

Ethics approval Ethic Review Board of the Centre de Recherche du Centre Hospitalier de l'Université de Montréal (CRCHUM, Montreal, Canada)

Provenance and peer review Not commissioned; externally peer reviewed for ethical and funding approval prior to submission.

Open Access This is an Open Access article distributed in accordance with the Creative Commons Attribution Non Commercial (CC BY-NC 4.0) license, which permits others to distribute, remix, adapt, build upon this work non-commercially, and license their derivative works on different terms, provided the original work is properly cited and the use is non-commercial. See: http://creativecommons.org/ licenses/by-nc/4.0/

(C) Article author(s) (or their employer(s) unless otherwise stated in the text of the article) 2018. All rights reserved. No commercial use is permitted unless otherwise expressly granted.

\section{REFERENCES}

1. Shaw JE, Sicree RA, Zimmet PZ. Global estimates of the prevalence of diabetes for 2010 and 2030. Diabetes Res Clin Pract 2010;87:4-14.

2. WHO. Global health estimates: deaths by cause, age, sex and country, 2000-2012. Geneva: World Health Organization, 2014.

3. WHO. Global status report on noncommunicable diseases 2014. Geneva: World Health Organization, 2012.

4. Morrish NJ, Wang SL, Stevens LK, et al. Mortality and causes of death in the WHO multinational study of vascular disease in diabetes. Diabetologia 2001;44(Suppl 2):S14-21.

5. WHO. Global status report on noncommunicable diseases 2010. Geneva: World Health Organization, 2011.

6. WHO. Global data on visual impairments 2010. Geneva: World Health Organization, 2012.

7. National Center for Health Statistics. Health, United States, 2014: With Special Feature on Adults Aged 55-64: Hyattsville, MD, 2015.

8. Pirmohamed M, James S, Meakin S, et al. Adverse drug reactions as cause of admission to hospital: prospective analysis of 18820 patients. BMJ 2004;329:15-19.

9. Nathan DM, Buse JB, Davidson MB, et al. Medical management of hyperglycemia in type 2 diabetes: a consensus algorithm for the initiation and adjustment of therapy: a consensus statement of the American Diabetes Association and the European Association for the Study of Diabetes. Diabetes Care 2009;32:193-203.

10. Esposito K, Chiodini P, Bellastella G, et al. Proportion of patients at $\mathrm{HbA1c}$ target $<7 \%$ with eight classes of antidiabetic drugs in type 2 diabetes: systematic review of 218 randomized controlled trials with 78945 patients. Diabetes Obes Metab 2012;14:228-33.

11. Hall HM, Banerjee S, McGuire DK. Variability of clopidogrel response in patients with type 2 diabetes mellitus. Diab Vasc Dis Res 2011;8:245-53. 
12. Manolopoulos VG, Ragia G, Tavridou A. Pharmacogenomics of oral antidiabetic medications: current data and pharmacoepigenomic perspective. Pharmacogenomics 2011;12:1161-91.

13. Holstein A, Plaschke A, Ptak M, et al. Association between CYP2C9 slow metabolizer genotypes and severe hypoglycaemia on medication with sulphonylurea hypoglycaemic agents. Br J Clin Pharmacol 2005;60:103-6.

14. Pacanowski MA, Hopley CW, Aquilante CL. Interindividual variability in oral antidiabetic drug disposition and response: the role of drug transporter polymorphisms. Expert Opin Drug Metab Toxicol 2008;4:529-44.

15. Aitken AE, Richardson TA, Morgan ET. Regulation of drugmetabolizing enzymes and transporters in inflammation. Annu Rev Pharmacol Toxicol 2006;46:123-49.

16. Abdel-Razzak Z, Loyer P, Fautrel A, et al. Cytokines down-regulate expression of major cytochrome P-450 enzymes in adult human hepatocytes in primary culture. Mol Pharmacol 1993;44:707-15.

17. Renton KW. Cytochrome $\mathrm{P} 450$ regulation and drug biotransformation during inflammation and infection. Curr Drug Metab 2004;5:235-43.

18. Cheng PY, Morgan ET. Hepatic cytochrome P450 regulation in disease states. Curr Drug Metab 2001;2:165-83.

19. Ryu JY, Song IS, Sunwoo YE, et al. Development of the Inje cocktail for high-throughput evaluation of five human cytochrome P450 isoforms in vivo. Clin Pharmacol Ther 2007;82:531-40.
20. Chainuvati S, Nafziger AN, Leeder JS, et al. Combined phenotypic assessment of cytochrome p450 1A2, 2C9, 2C19, 2D6, and $3 \mathrm{~A}, \mathrm{~N}$-acetyltransferase-2, and xanthine oxidase activities with the "Cooperstown 5+1 cocktail". Clin Pharmacol Ther 2003:74:437-47.

21. Christensen M, Andersson K, Dalén P, et al. The Karolinska cocktail for phenotyping of five human cytochrome P450 enzymes. Clin Pharmacol Ther 2003;73:517-28.

22. Frye RF, Matzke GR, Adedoyin A, et al. Validation of the fivedrug "Pittsburgh cocktail" approach for assessment of selective regulation of drug-metabolizing enzymes. Clin Pharmacol Ther 1997;62:365-76.

23. Turpault $\mathrm{S}$, Brian $\mathrm{W}$, Van Horn $\mathrm{R}$, et al. Pharmacokinetic assessment of a five-probe cocktail for CYPs 1A2, 2C9, 2C19, 2D6 and 3A. Br J Clin Pharmacol 2009;68:928-35.

24. Zhu B, Ou-Yang DS, Chen XP, et al. Assessment of cytochrome P450 activity by a five-drug cocktail approach. Clin Pharmacol Ther 2001;70:455-61.

25. Diczfalusy U, Miura J, Roh HK, et al. 4Beta-hydroxycholesterol is a new endogenous CYP3A marker: relationship to CYP3A5 genotype, quinine 3-hydroxylation and sex in Koreans, Swedes and Tanzanians. Pharmacogenet Genomics 2008;18:201-8.

26. Diczfalusy $\mathrm{U}$, Nylén $\mathrm{H}$, Elander $\mathrm{P}$, et al. 4 4 -Hydroxycholesterol, an endogenous marker of CYP3A4/5 activity in humans. $\mathrm{Br} \mathrm{J}$ Clin Pharmacol 2011;71:183-9. 